\title{
Symmetries of special 2-flags
}

\author{
Piotr Mormul and Fernand Pelletier
}

\begin{abstract}
This work is a continuation of authors' research interrupted in the year 2010. Derived are recursive relations describing for the first time all infinitesimal symmetries of special 2-flags (sometimes also misleadingly called 'Goursat 2-flags'). When algorithmized to the software level, they will give an answer filling in the gap in knowledge as of 2010: on one side the local finite classification of special 2-flags known in lengths not exceeding four, on the other side the existence of a continuous numerical modulus of that classification in length seven.
\end{abstract}

\section{Introduction}

The paper is devoted to 'special 2-flags', that is, strictly speaking, to rank 3 distributions generating special 2-flags. More particularly - to the symmetries of such distributions.

There circulates a widely acknowledged folk theorem (cf. section 4 in 23 . and p. 86 in [10]) saying that, outside the so-called stable range, distributions generic enough do not possess any nontrivial, even only local, symmetry. More to the point, in concrete classical classes of subbundles in the tangent bundle, like the ' 3,5 ' or ' 4,7 ' distributions, the (Lie) groups of symmetries are severely restricted in size: not bigger than 14-dimensional in the former (maximal in the flat case, when the Cartan tensor - 3] - vanishes; [10], p. 88 and [2], p. 456), and not bigger than 21-dimensional in the latter (maximal for the instanton distribution, [10, p. 90). And, naturally, likewise restricted in size are the Lie algebras of vector fields - infinitesimal symmetries (i.s. for short in all what follows). (They always form a Lie algebra due to the Jacobi identity.) While for the objects discussed in this work, by virtue of their rather stringent definition, the i. s.' algebras are infinite-dimensional. Much like it is the case for the 1flags, i.e., Goursat flags discussed here in length, in the guise of 'forerunners', in - still introductory - Sections 2 and 3. (The i. s.'s for Goursat structures are parametrized by one free function of three variables - a so-called contact hamiltonian.)

The purpose of this paper is to exhibit (for the first time) recursive relations which describe all i.s.'s of special 2-flags. In order to precise this context and give first some motivations, we start from 2 -flags of length 1 . That is, rank 3 distributions $D \subset T M, \operatorname{dim} M=5$ such that $D+[D, D]=T M$ (or, the same 
thing, $[D, D]=T M$; the first order Lie brackets generate all the remaining tangent directions; distribution is 'two-step'). Willy-nilly one enters the domain of the classical 'cinq variables' work [3]. It was shown there that every such two step $D$ possessed uniquely determined corank 1 subdistribution $F$ enjoying the property

$$
[F, F] \subset D
$$

(see equations (4) on p.121 in 3]). Cartan calls such an accompanying subdistribution $F$ le système covariant of [the Pfaffian system] $D$. Cartan firstly discerns a highly particular situation (a) when $[F, F]=F$ identically in the vicinity of a point. As a consequence, he infers that, in certain local coordinates $t, x^{0}, y^{0}, x^{1}, y^{1}, D$ gets description $d x^{0}-x^{1} d t=0=d y^{0}-y^{1} d t$. In contemporary terminology, such $D$ is, up to a local coordinate change, the classical Cartan distribution, or contact system, on the jet space $J^{1}(1,2)$ of the 1 -jets of functions $\mathbb{R}(t) \rightarrow \mathbb{R}^{2}(x, y)$, with $x^{1}=\frac{d x^{0}}{d t}$ and $y^{1}=\frac{d y^{0}}{d t}$. Its corank 1 covariant subdistribution $F$ (reiterating, involutive in situation (a)!) is in these coordinates just $\operatorname{span}\left(\frac{\partial}{\partial x^{1}}, \frac{\partial}{\partial y^{1}}\right)$. In all what follows we will skip the symbol 'span' before a set of vector field generators.

By far more interesting is Cartan's situation (b) $[F, F]=D$ in the vicinity of a given point 1 The covariant object $F$ has then its 'curvature' and $D$ is retrievable from $F$ alone. We note that situation (b) is extremely rich geometrically and hides a functional modulus (one function of five variables) of the local classification of ' 3,5 ' distributions with respect to the diffeos of base manifold.

We say that a general such $D$ (with no extra information as to (a) or (b)) generates a 2 -flag of length 1 , while a $D$ with its covariant system $F$ involutive generates a special 2 -flag of length 1 .

Therefore, the adjective 'special' in length 1 locally means nothing but 'jet-like'. How does it look like in bigger lengths/higher jets?

Let us analyze the contact system $D$ on a concrete jet space $J^{r}(1,2)=: M$ with $r \geq 1$. The main observation is that the sequence of modules of vector fields - consecutive Lie squares of $D$,

$$
T M=D^{0} \supset D^{1} \supset D^{2} \supset \cdots \supset D^{r-1} \supset D^{r},
$$

where $D^{r}=D$ and $\left[D^{j}, D^{j}\right]=D^{j-1}$ for $j=r, r-1, \ldots, 2,1$, grows in ranks regularly by two: $3,5,7, \ldots, 2 r+1,2(r+1)+1=\operatorname{dim} M$ independently of the underlying points in $M$. (Pay attention to the indexation, which starts with the biggest index $r$, following the notation put forward in [11.) The reason is that in passing from $D^{j}$ to $D^{j-1}$ one forgets about the $j$-th order derivatives, so that

$$
D^{j-1}=\left(D^{j}, \frac{\partial}{\partial x^{j}}, \frac{\partial}{\partial y^{j}}\right)
$$

Therefore, all these modules of vector fields are actually distributions which together form a 2-flag of length $r$ on $M$. Let us scrutinize the members of this

${ }^{1}$ situations (a) and (b) do not exhaust all possibilities of the local behaviour of $F$; Elie Cartan used to be interested in clear situations only 
flag.

The natural coordinates in $J^{r}(1,2)$ are $t, x^{0}, y^{0}, x^{1}, y^{1}, \ldots, x^{r}, y^{r}$, where $x^{j}=$ $\frac{d x^{j-1}}{d t}, y^{j}=\frac{d y^{j-1}}{d t}$ for $j=1,2, \ldots, r$. In these coordinates the one before last member $D^{1}$ in (2) has a Pfaffian equations' description $d x^{0}-x^{1} d t=0=$ $d y^{0}-y^{1} d t$, hence it manifestly contains a corank 1 involutive subdistribution

$$
F:=\left(\frac{\partial}{\partial x^{j}}, \frac{\partial}{\partial y^{j}} ; 1 \leq j \leq r\right) .
$$

Likewise, the next smaller member $D^{2}$ has description

$$
d x^{0}-x^{1} d t=d y^{0}-y^{1} d t=0=d x^{1}-x^{2} d t=d y^{1}-y^{2} d t,
$$

hence contains a corank 1 involutive subdistribution

$$
\left(\frac{\partial}{\partial x^{j}}, \frac{\partial}{\partial y^{j}} ; 2 \leq j \leq r\right) .
$$

The key point is that the latter happens to be the Cauchy-characteristic module of $D^{1}$, denoted by $L\left(D^{1}\right)$ as in 11, 2 This pattern replicates itself all the way down the flag. The Pfaffian systems describing $D^{j}$ gradually get larger sets of Pfaffian equations' generators, while the Cauchy-characteristic modules get (with a shift in indices!) thinner. In fact, for $1 \leq j<r$,

$$
L\left(D^{j}\right)=\left(\frac{\partial}{\partial x^{s}}, \frac{\partial}{\partial y^{s}} ; j+1 \leq s \leq r\right)
$$

sits inside $D^{j+1}$ as a corank 1 subdistribution. For instance $L\left(D^{r-1}\right)$ is a field of planes $\left(\frac{\partial}{\partial x^{r}}, \frac{\partial}{\partial y^{r}}\right)$ sitting inside a field of 3-spaces $D^{r}$, while $L\left(D^{r}\right)=(0)$. Moreover all these geometric objects nicely fit together into Sandwich Diagram, so called after a similar (if not identical) diagram assembled for Goursat distributions, or 1-flags, in [11.

$$
\begin{array}{ccccccccccccc}
T M=D^{0} & \supset & D^{1} & \supset & D^{2} & \supset & D^{3} & \cdots & D^{r-1} & \supset & D^{r} & & \\
& & \cup & & \cup & & \cup & & \cup & & \cup & \cup \\
& F & \supset & L\left(D^{1}\right) & \supset & L\left(D^{2}\right) & \cdots & L\left(D^{r-2}\right) & \supset & L\left(D^{r-1}\right) & \supset & L\left(D^{r}\right)=0 .
\end{array}
$$

All vertical inclusions in the diagram are of codimension one, while all (drawn, we do not mean superpositions of them) horizontal inclusions are of codimension 2. The squares built by these inclusions can, indeed, be perceived as certain 'sandwiches'. For instance, in the utmost left sandwich $F$ and $D^{2}$ are as if fillings, while $D^{1}$ and $L\left(D^{1}\right)$ constitute the covers (of dimensions differing by 3 , one has to admit). At that, the sum $2+1$ of codimensions, in $D^{1}$, of $F$ and $D^{2}$

\footnotetext{
${ }^{2}$ For $D$-a distribution, $L(D)$ is, by definition, the module of Cauchy-characteristic vector fields with values in $D$ infinitesimally preserving $D$. That module is automatically (the Jacobi identity) closed under the Lie bracket. It is noteworthy that for all the particular distributions $D$ occurring in the present work, $L(D) \subset D$ is always not just a module included in $D$, but an involutive subdistribution of $D$ of corank 2 (or 3 ) when $m=1$ (or 2).
} 
equals the dimension of the quotient space $D^{1} / L\left(D^{1}\right)$, so that it is natural to ask how the 2-dimensional plane $F / L\left(D^{1}\right)$ and the line $D^{2} / L\left(D^{1}\right)$ are mutually positioned in $D^{1} / L\left(D^{1}\right)$ : do they intersect regularly, or else the plane subsumes line:3 Clearly, that question imposes by itself in further sandwiches 'indexed' by the upper right vertices $D^{3}, D^{4}, \ldots, D^{r}$, as well.

This question has a trivial answer for the Cartan distribution $D=D^{r}$ analyzed above (all intersections are regular when $r \geq 2$ ). Yet a more pertinent question would be the following.

Assume the existence of Sandwich Diagram with all its above-listed dimensions, inclusions, involutivenesses and call such rank 3 distributions $D^{r}$ generating special 2-flags of length $r$. Are then those $D^{r}$ locally 'jet-like', that is - locally equivalent to the Cartan contact distribution on $J^{r}(1,2)$ ?

For $r=1$, we reiterate, yes ([3]), but for $r=2$ already not. There suffices to seemingly slightly modify system (44) to

$$
d x^{0}-x^{1} d t=d y^{0}-y^{1} d t=0=d t-x^{2} d x^{1}=d y^{1}-y^{2} d x^{1} .
$$

This rank 3 distribution on $\mathbb{R}^{7}$ does generate a special 2-flag of length 2 , yet is not locally equivalent to the 'jet-like' one around every point with $x^{2}=0$ (cf. [16, Prop. 1 (iii)). The argument there has been that the object (5) has at points $x^{2}=0$ the small growth vector $(3,5,6,7)$, while the contact system on $J^{2}(1,2)$ has everywhere the small growth vector $(3,5,7)$. Another, possibly even simpler argument is that at points $x^{2}=0$ there is no regular intersection in the only sandwich existing in that length: the line $D^{2} / L\left(D^{1}\right)$ collapses onto the plane $F / L\left(D^{1}\right)$, while the analogous line for (4) collapses nowhere.

Therefore it follows that the local theory of special multi-flags is not 'void' in the sense of boling down to the contact systems on the jet spaces for curves. In fact, this theory is already fairly rich and still developing, including this work.

Let us reiterate the importance of 'special' for 2-flags to be tractable (and the same for multi-flags in general). Special, by the way of Sandwich Diagram, brings in so much stiffness as to result in the local models with numerical moduli only, no functional ones. While functional moduli, by simple and widely known dimension counts (cf., for inst., section 3 in [23]) are a commonplace in the local geometry of subbundles in tangent bundles. Even the already mentioned paper [3] about 2-flags of length 1 is not yet fully understood! On the other side, the initial departing models for us - contact systems on the jet spaces - are nowadays viewed as just the simplest 'baby' realizations of the special multi-flags.

Attention. This theory is even more neat in that it does not necessitate a definition via Sandwich Diagram as such. For it follows from the important works 21, 1] that, upon assuming only the properties of the upper row in Sandwich

\footnotetext{
${ }^{3}$ this suffices to tell (5) from (4) in what follows below

${ }^{4}$ The small growth vector of a distribution $D$ at a point $p$ is the sequence of integer numbers $\left(\operatorname{dim} V_{j}(p)\right)_{j \geq 1}$, where $V_{1}=D, V_{j+1}=V_{j}+\left[D, V_{j}\right]$, which ends on the first biggest entry.
} 
Diagram and the existence of a whatever corank one involutive subdistribution $F$ in $D^{1}$, one automatically gets Sandwich Diagram in its entirety!

In fact, (i) such an $F$ is then unique, (ii) for $j=1,2, \ldots, r-1$ there holds

$$
L\left(D^{j}\right)=D^{j+1} \cap F,
$$

(iii) $L\left(D^{r}\right)=(0)$ and (iv) the $L\left(D^{j}\right)$ 's are corank 1 subdistributions in $D^{j+1}$, so that Sandwich Diagram entirely holds.

Now that the focus is again on Sandwich Diagram, the ongoing question bears on the local geometry in the sandwiches 'indexed' by the upper right vertices $D^{2}, D^{3}, \ldots, D^{r}$. It naturally opens the way towards singularities. The

first step in that direction is a [fairly raw] stratification of germs of special 2-flags into so-called sandwich classes. The second is further partitioning of sandwich classes into singularity classes (see section 4.1).

\section{Kumpera-Ruiz watching glasses for Goursat distributions}

In order to gently introduce the reader to the main techniques of the paper, we present in this section a test case - derive the formulas for the infinitesimal symmetries of Goursat distributions which generate 1-flags. This will be instrumental during the presentation of similar things to-be-derived for [special] 2-flags in paper's subsequent sections.

Recalling, a rank 2 distribution on a manifold $M$ is Goursat when the tower of its consecutive Lie squares, understood as modules of vector fields, consist uniquely of regular distributions of ranks $3,4,5, \ldots$ until $n=\operatorname{dim} M$.

With no loss in generality, Goursat distributions understood locally live on the stages of Goursat Monster Tower (GMT for short), by some authors called alternatively Semple Tower. The stages have been denoted in [12] by $\mathbb{P}^{r} \mathbb{R}^{2}$, $r \geq 2$. (On the stage $\mathbb{P}^{r} \mathbb{R}^{2}$ there lives a Goursat distribution of corank $r$.) The best glasses to watch Goursat distributions are Kumpera-Ruiz coordinates (KR for short), 9]. Those are semi-global sets of coordinates (their domain of definition is always dense in a given tower's stage) which critically depend on the strata of a most natural stratification of any given stage $\mathbb{P}^{r} \mathbb{R}^{2}$ - so-called Kumpera-Ruiz classes, KR-classes for short, see [11, p. 466. They exist in $\mathbb{P}^{r} \mathbb{R}^{2}$ in number $2^{r-2}$ and are univocally labelled by the words of length $r$ over the alphabet $\{1,2\}$, with two first letters always $1: 1.1 . i_{3} . i_{4} \ldots i_{r}$. (In [1] they were originally labelled by the subsets $I \subset\{3,4, \ldots, r\}$, a given $I$ consisting of the indices $j$ such that $i_{j}=2$.) The KR classes are the main tool in the introductory part of our paper. Their generalizations for special 2-flags, socalled singularity classes, will play a similar role in the main part of the present contribution from Section 4 onwards.

To each KR-class attached are handy coordinates making that class visible. More precisely, due to the particular topology of the two lowest Monster's stages 
$\mathbb{P}^{1} \mathbb{R}^{2}$ and $\mathbb{P}^{2} \mathbb{R}^{2}$, they both are unions of pairs of open dense subsets, $\mathbb{P}^{1} \mathbb{R}^{2}=$ $U_{1} \cup U_{2}$ and $\mathbb{P}^{2} \mathbb{R}^{2}=V_{1} \cup V_{2}$ such that, for each KR-class $\mathcal{C}=1.1 . i_{3} . i_{4} \ldots i_{r}$ and indices $j, k \in\{1,2\}$

$$
\mathcal{C} \cap \pi_{r, 1}^{-1}\left(U_{j}\right) \cap \pi_{r, 2}^{-1}\left(V_{k}\right)
$$

sits in the domain of [Kumpera-Ruiz] coordinates $x_{1}, x_{2}, \ldots, x_{r+2}$ produced precisely for the data $\mathcal{C}, j, k$.

Remark 1. The open dense sets $U_{j}$ and $V_{k}$ are related to the ways the Darboux theorem (in the contact $3 \mathrm{D}$ manifold $\mathbb{P}^{1} \mathbb{R}^{2}$ ) and Engel theorem (in the Engel $4 \mathrm{D}$ manifold $\mathbb{P}^{2} \mathbb{R}^{2}$ ) come into effect. In those coordinates

$$
\Delta^{r}=\left(Y[r], \partial_{r+2}\right)
$$

where, in what follows, $\partial_{j}=\frac{\partial}{\partial x^{j}}$ and $Y[r]$ is a polynomial vector field defined recursively as follows.

Initially $Y[1]=\partial_{1}+x^{3} \partial_{2}$ and $Y[2]=Y[1]+x^{4} \partial_{3}$. When, for $j \geq 3, Y[j-1]$ is already defined and $i_{j}=1$, then $Y[j]=Y[j-1]+x^{j+2} \partial_{j+1}$. In the opposite case of $i_{j}=2$ one puts $Y[j]=x^{j+2} Y[j-1]+\partial_{j+1}$. The eventual vector field $Y[r]$ in (7) is, therefore, polynomial of degree $(1+$ the \# of letters 2 in the code of $\mathcal{C}$ ). That degree is maximal (and equal $r-1$ ) when the underlying KR-class is 1.1.2.2 ..2 $(r-2$ letters 2 past the initial segment 1.1).

Remark 2. Whenever $i_{j}=2$ in the code of $\mathcal{C}$, the variable $x^{j+2}$ brought in at the $j$-th step of the above procedure vanishes at points of (6). This is a key property of the polynomial visualisations of Goursat distributions put forward in 9 .

The KR-classes are invariant with respect to the local diffeomorphisms of Monster's relevant stages. They are only very rough approximations to local models (local normal forms). To really approach the orbits, one would need to know the (pseudo-)groups of i. s.'s of the structures $\Delta^{r}$ living on $\mathbb{P}^{r} \mathbb{R}^{2}$. Those groups are infinite-dimensional, for they consist of due prolongations of the contact vector fields which preserve the contact structure $\Delta^{1}$. In order to see them, one puts on, no wonder, KR-glasses. That is, works and computes in chosen KR-coordinates.

\section{Infinitesimal symmetries of Goursat flags}

From now on we assume that KR-coordinates, pertinent for a fixed KR-class in length $r$, have been picked and frozen. In these coordinates, every concrete i.s. writes down as $\mathcal{Y}_{f}=\sum_{i=1}^{r} F^{i} \partial_{i}$, where the first three components are functions of one (smooth) generating function in three variables, say $f\left(x^{1}, x^{2}, x^{3}\right)$ :

$$
F^{1}=-f_{3}, \quad F^{2}=f-x^{3} f_{3}, \quad F^{3}=f_{1}+x^{3} f_{2},
$$

and the remaining components are other, more complicated functions of $f$ depending on the KR-class in question, as will be recalled in what follows. Such 
one free function $f$ is called a contact hamiltonian; the infinite dimensionality of the symmetry pseudogroup is visible.

When a vector field $\mathcal{Y}_{f}$ preserves infinitesimally the Goursat $\Delta^{r}$, the truncations of $\mathcal{Y}_{f}$ do infinitesimally preseve all the earlier (older) Goursat structures showing up in the process of building up $\Delta^{r}$. In fact, each component $F^{s}$, $s=4,5, \ldots, r+2$, depends only on the variables $x^{1}, x^{2}, \ldots, x^{s}$ and

$$
\left[\sum_{i=1}^{j+2} F^{i} \partial_{i}, \Delta^{j}\right] \subset \Delta^{j}
$$

for $j=1,2, \ldots, r$, where $\Delta^{j}=\left(Y[j], \partial_{j+2}\right)$, as in (7). This technically central statement is well-known in the theory of Goursat structures, compare for instance Proposition 1 in [14. Besides, this triangle nature of the i.s.'s of Goursat structures will be clearly visible in the recurrences that are produced below. The first prolongation of an infinitesimal contactomorphism $\sum_{i=1}^{3} F^{i} \partial_{i}$ is $\sum_{i=1}^{4} F^{i} \partial_{i}$, and the new component is univocally determined by the previous ones,

$$
F^{4}=Y[2] F^{3}-x^{4} Y[2] F^{1},
$$

compare p. 222 in [14]. Reiterating, the components $F^{1}$ and $F^{3}$ entering formula (10) depend on the first three variables, and the field $\stackrel{2}{Y}$ differentiates them accordingly. In the outcome, the component $F^{4}$ depends on the first four variables, and so it goes further on. (This formula is, in fact, subsumed in the line of derivations that follow. It is given here prior to more involved relations that depend already on the KR-class underlying the KR coordinates in use.)

We work with a fixed class $\mathcal{C}=1.1 . i_{3} . i_{4} \ldots i_{r}$ and with a fixed letter $i_{j}$ in its code, $j \geq 3$. In order to word the recurrences governing the i.s.'s of $\mathcal{C}$, we need a

Definition of $s(j)$ for Goursat flags. There can, or cannot, be letters 2 before the letter $i_{j}$.

$s(j):= \begin{cases}0, & \text { when there is no letter } 2 \text { in the code of } \mathcal{C} \text { before } i_{j}, \\ s, & \text { the farthest position of a letter } 2 \text { before } i_{j} \text { is } s, \text { in the opposite case } .\end{cases}$

Theorem 1 ([13]) Suppose that the components $F^{1}, F^{2}, \ldots, F^{j+1}, j \geq 3$, of an infinitesimal symmetry $\mathcal{Y}_{f}$ of $\Delta^{r}$ in the vicinty of a KR-class $\mathcal{C}=$ 1.1. $i_{3} . i_{4} \ldots i_{r}$ are already known. When $i_{j}=1$, then

$$
F^{j+2}= \begin{cases}Y[j] F^{j+1}-x^{j+2} Y[2] F^{1}, & \text { when } s(j)=0, \\ Y[j] F^{j+1}-x^{j+2} Y[s(j)] F^{s(j)+1}, & \text { when } s(j) \geq 3 .\end{cases}
$$

When $i_{j}=2$, then

$$
F^{j+2}= \begin{cases}x^{j+2}\left(Y[2] F^{1}-Y[j] F^{j+1}\right), & \text { when } s(j)=0, \\ x^{j+2}\left(Y[s(j)] F^{s(j)+1}-Y[j] F^{j+1}\right), & \text { when } s(j) \geq 3 .\end{cases}
$$


Note before the proof that, on the whole, there are $2^{j-2}$ versions of the formulas for the component function $F^{j+2}$, all of them encoded in this theorem. For that many KR-classes exist in length $j$. Those formulas are polynomials in the $x$ variables, of growing degrees, with coefficients - partials (of growing orders) of a contact hamiltonian $f$.

The original proof of this theorem occupied full four pages in [13]. Now we are going to re-prove it in a much shorter manner. Then this new method will be generalized and applied to the 2-flags' case in the sections that follow.

To begin with, the truncation of the field $\mathcal{Y}_{f}$ to the Monster level $j, \sum_{i=1}^{j+2} F^{i} \partial_{i}$, preserves the Goursat structure $\Delta^{j}$, as is noted already in (9). Implying, that

$$
\left[\sum_{i=1}^{j+2} F^{i} \partial_{i}, Y[j]\right]=a_{j} Y[j]+b_{j} \partial_{j+2}
$$

for certain unspecified functions $a_{j}$ and $b_{j}$ of variables $x^{1}, \ldots, x^{j+2}$.

Now we consider the situation $i_{j}=1$. Remembering the construction of the field $Y[j]$ when the underlying KR-class is $\mathcal{C}$ :

- when $s(j)=0$, the first $\left(\partial_{1}\right)$ component on the LHS of (11) is $-Y[2] F^{1}$. And

$\bullet \bullet$ when $s(j) \geq 3$, the $(s(j)+1)$-st component on the LHS of (11) is $-Y[s(j)] F^{s(j)+1}$. So

$$
a_{j}= \begin{cases}-Y[2] F^{1}, & \text { when } s(j)=0, \\ -Y[s(j)] F^{s(j)+1}, & \text { when } s(j) \geq 3 .\end{cases}
$$

One compares now the $(j+1)$-st components on the both sides of (11), obtaining

$$
F^{j+2}-Y[j] F^{j+1}=a_{j} x^{j+2} .
$$

Substituting on the RHS here the expressions (12) in due order, one gets closed form formulas for the $\partial_{j+2}$ - component function $F^{j+2}$, as invoiced in the theorem. As for the coefficient function $b_{j}$ in (11), it is - here and in what follows later - ascertained last, after finding out $F^{j+2}$.

In the situation $i_{j}=2$ the arguments differ only technically. Now, regardless of the value of $s(j)$, the coefficient $a_{j}$ can be extracted from (11) at the level $\partial_{j+1}$ : on the LHS it is $-Y[j] F^{j+1}$, and it is a plain $a_{j}$ on the RHS. Hence

$$
a_{j}=-Y[j] F^{j+1} .
$$

Then, no wonder, one compares the coefficients in (11) at: $\partial_{1}$, when $s(j)=$ 0 , or else at $\partial_{s(j)+1}$, when $s(j) \geq 3$. In the former case one fetches on the LHS the quantity $F^{j+2}-x^{j+2} Y[2] F^{1}$. In the latter, the quantity $F^{j+2}-$ $x^{j+2} Y[s(j)] F^{s(j)+1}$.

At the same time one fetches $a_{j} x^{j+2}$ on the RHS, just irrelevantly of the case in question. That is, accounting for (13),

$$
F^{j+2}-x^{j+2} Y[2] F^{1}=-Y[j] F^{j+1} x^{j+2}
$$


(when $s(j)=0$ ), or else

$$
F^{j+2}-x^{j+2} Y[s(j)] F^{s(j)+1}=-Y[j] F^{j+1} x^{j+2}
$$

(when $s(j) \geq 3$ ). A closed form formula for $F^{j+2}$, invoiced earlier, follows immediately. Only then the $b_{j}$ coefficient is got hold of. In order to conclude that the ascertained vector field actually is a symmetry of $\Delta^{r}$ one observes that, in each of the underlying $2^{r-2}$ situations,

$$
\left[\sum_{i=1}^{r+2} F^{i} \partial_{i}, \partial_{r+2}\right]=\left(-\partial_{r+2} F^{r+2}\right) \partial_{r+2},
$$

because only its last component function $F^{r+2}$ depends on the last variable $x^{r+2}$. Theorem 1 is now proved.

\section{Special 2-flags: a basic toolkit}

Special 2-flags constitute a natural follow-up to Goursat flags. The latter compactify (in certain precise sense) the contact Cartan distributions on the jet spaces $J^{r}(1,1)$, while the former do the same with respect to the jet spaces $J^{r}(1,2)[5$

Sequences of Cartan prolongations of rank 3 distributions are the key players in producing (only locally, though) virtually all rank 3 distributions generating special 2-flags. There quickly emerges an immense tree of singularities of positive codimensions, all of them adjoining the unique open dense Cartan-like strata.

While the local classification problem is well advanced for the Goursat flags, most notably after the work [12, it is much less advanced for special 2-flags (or, more generally, for special multi-flags). It was first attacked in [8], then, in the chronological order, in: [15, [16], 22], 21, 17, [1, and [18. After the year 2010 researchers were aiming at defining various invariant stratifications in the spaces of germs of special multi-flags: [19, [6], [5], 20]. The actual state of the art is reflected in a recent summarizing work [4]. The works [19] and [20] stand out due to a kinematical interpretation of the special 2-flags developed in them. Namely, a model of an articulated arm in the 3D space with an engine, or a spacecraft with attached string of satellites. The singularities related to various possible distributions of right angles between neighbouring segments are already well understood and encoded. However, the issue of constructing a kinematics-driven fine stratification analogous to Jean's one [7] of the car + trailers systems (modelling 1-flags) in terms of Jean's critical angles, is not yet solved. In particular, a faithful expression of the classes in the benchmark work 4, in the terms of an articulated arm in 3D space, seems to be out of reach. The issue mentioned above is, most likely, equivalent to that of computing all small growth vectors for distributions generating special 2-flags.

\footnotetext{
${ }^{5}$ Some researchers, e.g. in [5], use, instead of 'special multi-flags' a somehow misleading synonym 'Goursat multi-flags'.
} 
In the work [18] there was completed only the classification of special 2flags in lengths not exceeding 4. At that time the machinery of infinitesimal symmetries for those objects was far from being assembled and the techniques in use were rather disparate. This notwithstanding, the precise number (34) of local equivalence classes of special 2-flags in length 4 was ascertained there (cf. the table below).

The driving force of the present work are the singularity classes (in the occurrence - of special 2-flags) known for 15 years already. They are technically most important for our purposes and results. We briefly recall their construction in the next section. For reader's convenience, here is the table of cardinalities of singularity classes, RV classes of Castro et al [4, and classes of the local equivalence of the special 2-flags, in function of flag's lengths not exceeding 7:

\begin{tabular}{|c||c|c||c|}
\hline length & \# sing classes & \# RV classes & \# orbits \\
\hline \hline 2 & 2 & 2 & 2 \\
\hline 3 & 5 & 6 & 7 \\
\hline 4 & 14 & 23 & 34 \\
\hline \hline 5 & 41 & 98 & $?$ \\
\hline 6 & 122 & 433 & $? ?$ \\
\hline 7 & 365 & 1935 & $\infty$ \\
\hline
\end{tabular}

Question. How to partition a given singularity class of special 2-flags into (much finer!) RV classes of [4] And, all the more so, for special $m$-flags, $m>2$ ?!

\subsection{Singularity classes of [germs of] special 2-flags refining the sandwich classes}

We first divide all existing germs of special 2-flags of length $r$ into $2^{r-1}$ pairwise disjoint sandwich classes in function of the geometry of the distinguished spaces in the sandwiches (at the reference point for a germ) in Sandwich Diagram on p. 3 , and label those aggregates of germs by words of length $r$ over the alphabet $\{1, \underline{2}\}$ starting (on the left) with 1 , having the second cipher 2 iff $D^{2}(p) \subset F(p)$, and for $3 \leq j \leq r$ having the $j$-th cipher $\underline{2}$ iff $D^{j}(p) \subset L\left(D^{j-2}\right)(p)$. More details about the sandwich classes are given in section 1.2 in [18.

This construction puts in relief possible non-transverse situations in the sandwiches. For instance, the second cipher is $\underline{2}$ iff the line $D^{2}(p) / L\left(D^{1}\right)(p)$ is not transverse, in the space $D^{1}(p) / L\left(D^{1}\right)$, to the codimension one subspace $F(p) / L\left(D^{1}\right)(p)$, and similarly in further sandwiches. This resembles very much the KR-classes of Goursat germs constructed in 11]. In length $r$ the number of sandwiches has then been $r-2$ (and so the \# of KR classes $2^{r-2}$ ). For 2 -flags the number of sandwiches is $r-1$ because the covariant distribution of $D^{1}$ comes into play and gives rise to one additional sandwich.

Passing to the main construction underlying our present contribution, we refine further the singularities of special 2-flags and recall from [15] how one passes from the sandwich classes to singularity classes. In fact, to any germ $\mathcal{F}$ of a special 2-flag associated is a word $\mathcal{W}(\mathcal{F})$ over the alphabet $\{1,2,3\}$, called 
the 'singularity class' of $\mathcal{F}$. It is a specification of the word 'sandwich class' for $\mathcal{F}$ (this last being over, reiterating, the alphabet $\{1, \underline{2}\}$ ) with the letters $\underline{2}$ replaced either by 2 or 3 , in function of the geometry of $\mathcal{F}$.

In the definition that follows we keep fixed the germ of a rank-3 distribution $D$ at $p \in M$, generating on $M$ a special 2-flag $\mathcal{F}$ of length $r$.

Suppose that in the sandwich class $\mathcal{C}$ of $D$ at $p$ there appears somewhere, for the first time when reading from the left to right, the letter $\underline{2}=j_{m}\left(j_{m}\right.$ is, as we know, not the first letter in $\mathcal{C}$ ) and that there are in $\mathcal{C}$ other letters $\underline{2}=j_{s}, m<s$, as well. We will specify each such $j_{s}$ to one of the two: 2 or 3 . (The specification of that first $j_{m}=\underline{2}$ will be made later and will be trivial.) Let the nearest $\underline{2}$ standing to the left to $j_{s}$ be $\underline{2}=j_{t}, m \leq t<s$. These two 'neighbouring' letters $\underline{2}$ are separated in $\mathcal{C}$ by $l=s-t-1 \geq 0$ letters 1 .

The gist of the construction consists in taking the small flag of precisely original flag's member $D^{s}$,

$$
D^{s}=V_{1} \subset V_{2} \subset V_{3} \subset V_{4} \subset V_{5} \subset \cdots,
$$

$V_{i+1}=V_{i}+\left[D^{s}, V_{i}\right]$, then focusing precisely on this new flag's member $V_{2 l+3}$. Reiterating, in the $t$-th sandwich, there holds the inclusion: $F(p) \supset D^{2}(p)$ when $t=2$, or else $L\left(D^{t-2}\right)(p) \supset D^{t}(p)$ when $t>2$. This serves as a preparation to our punch line (cf. [15, 17]).

Surprisingly perhaps, specifying $j_{s}$ to 3 goes via replacing $D^{t}$ by $V_{2 l+3}$ in the relevant sandwich inclusion at the reference point. That is to say, $j_{s}=\underline{2}$ is being specified to 3 iff $F(p) \supset V_{2 l+3}(p)$ (when $t=2$ ) or else $L\left(D^{t-2}\right)(p) \supset V_{2 l+3}(p)$ (when $t>2$ ) holds.

In this way all non-first letters $\underline{2}$ in $\mathcal{C}$ are, one independently of another, specified to 2 or 3 . Having that done, one simply replaces the first letter $\underline{2}$ by 2 , and altogether obtains a word over $\{1,2,3\}$. It is the singularity class $\mathcal{W}(\mathcal{F})$ of $\mathcal{F}$ at $p$.

Example. In length 4 there exist the following fourteen singularity classes: 1.1.1.1, 1.1.1.2; 1.1.2.1, 1.1.2.2, 1.1.2.3; 1.2.1.16 1.2.1.2, 1.2.1.3, 1.2.2.1, 1.2.2.2, 1.2.2.3, 1.2.3.1, 1.2.3.2, 1.2.3.3. (cf. the table on p. 9 ).

(In length $r$ the \# of singularity classes is $\frac{1}{2}\left(3^{r-1}+1\right)$; the codimension of a class equals the \# of 2's plus twice the \# of 3 's in the relevant code word.)

\subsection{New approach in the classification problem.}

A new (2017) approach to the local classification of flags starts with the effective (recursive) computation of all infinitesimal symmetries of special 2-flags, extending the work done (in [13]) for 1-flags, reproduced with essential shortcuts in Section 3 above. The recursive patterns depend uniquely on the singularity classes of special 2-flags recapitulated above. Those classes are coarser, yes, but much fewer - see the table preceding section 4.1 - than the RV classes summarized (and so neatly systematized) in [4].

\footnotetext{
${ }^{6}$ see section 7.2 for more information about precisely this class
} 
Polynomial visualisations of objects in the singularity classes, recalled in Section 5, are called EKR's (Extended Kumpera-Ruiz). They 'only' feature finite families of real parameters. Then the local classification problem is rephrased as a search for ultimate normalizations among such families of parameters. Having an explicit hold of the infinitesimal symmetries at each prolongation step, the freedom in varying those parameters will be ultimately reduced to solvability questions of (typically huge) systems of linear equations.

In fact, that linear algebra involves only partial derivatives, at the reference point, of the first three components of a given infinitesimal symmetry which are completely free functions of 3 variables (Lemma 1). Keeping the preceding part of a [germ of a] flag in question frozen imposes a sizeable set of linear conditions upon those derivatives up to certain order. Then some other linear combinations of them appear, or not, to be free - just in function of the local geometry of the prolonged distribution. This, in short, would determine the scope of possible normalizations in the new (emerging from prolongation) part of EKR's. See sections 7.1 and 7.2 below for more details.

\section{$5 \quad$ EKR glasses for singularity classes of special 2-flags}

According to section 4.1 the singularity classes of special 2-flags of length $r$ are univocally encoded by words of length $r$ over the alphabet $\{1,2,3\}$ such that: - the first letter is always 1 , and - a letter 3 , if any, must be preceded by a letter 2. That is to say, abusing notation a bit, for a singularity class $\mathcal{C}=1 . i_{2} . i_{3} \ldots i_{r}$ over $\{1,2,3\}$, a letter $i_{2}$ is either 1 or 2 , and a letter 3 may show up not earlier than at the 3rd position, provided there is a letter 2 before it. (We call it, especially in the wider context of special $m$-flags with arbitrary $m$, 'the least upward jumps rule', cf. [16.)

For instance, $\mathcal{C}=1.2 .3$ is a legitimate singularity class of length 3 (and, in the occurrence, of codimension three in the pertinent Monster's stage No 3).

For each such $\mathcal{C}$ we are going to introduce coordinates, in the number of $2 r+3$,

$$
t, x^{0}, y^{0}, x^{1}, y^{1}, \ldots, x^{r}, y^{r},
$$

in which the special rank 3 distribution - let us, from now on, call it $\Delta^{r}$ again - living on the Monster's $r$-th stage becomes visible. Those coordinates, we reiterate it, will sensitively depend on a class $\mathcal{C}$. In fact, skipping the geometric and also Lie-algebra-related arguments presented in detail in [17, within the domain of those coordinates (subsuming the class $\mathcal{C}$ ),

$$
\Delta^{r}=\left(Z[r], \partial_{x^{r}}, \partial_{y^{r}}\right),
$$

where the vector field $Z[r]$ is being defined recursively, shadowing step after step the code $1 . i_{2} \cdot i_{3} \ldots i_{r}$ of $\mathcal{C}$. The beginning of recurrence is $Z[1]=\partial_{t}+x^{1} \partial_{x^{0}}+$ $y^{1} \partial_{y^{0}}$, and, quite simply, $\Delta^{1}=\left(Z[1], \partial_{x^{1}}, \partial_{y^{1}}\right)$ on $\mathbb{R}^{5}\left(t, x^{0}, y^{0}, x^{1}, y^{1}\right)$. 
In the recurrence step one assumes description (15) known for $j-1$ in the place of $r$, where $1 \leq j-1 \leq r-1$, and puts

$$
Z[j]= \begin{cases}Z[j-1]+x^{j} \partial_{x^{j-1}}+y^{j} \partial_{y^{j-1}}, & \text { when } i_{j}=1, \\ x^{j} Z[j-1]+\partial_{x^{j-1}}+y^{j} \partial_{y^{j-1}}, & \text { when } i_{j}=2, \\ x^{j} Z[j-1]+y^{j} \partial_{x^{j-1}}+\partial_{y^{j-1}}, & \text { when } i_{j}=3 .\end{cases}
$$

In the end of this recurrence (for $j=r$ ) the description (15) tout courte is arrived at, on $\mathbb{R}^{2 r+3}$ in the variables (14). The final first vector field' generator $Z[r]$ is a, possibly deeply involved (in function of $\mathcal{C}$ ), polynomial vector field.

Our objective is to ascertain all infinitesimal symmetries $\mathcal{Y}$ of (15) in the vicinity of any particular class $\mathcal{C}$. They will, no wonder, sensitively depend on $\mathcal{C}$, too. Let us have such $\mathcal{Y}$ expanded in EKR coordinates chosen for $\mathcal{C}$ :

$$
\mathcal{Y}=A \partial_{t}+B \partial_{x^{0}}+C \partial_{y_{0}}+\sum_{s=1}^{r}\left(F^{s} \partial_{x^{s}}+G^{s} \partial_{y^{s}}\right) .
$$

The first key property (needed later) is

Lemma 1 The component functions $A, B, C$ in (17) depend only on the variables $t, x^{0}, y^{0}$.

Proof of Lemma 1. The reason is that, whatever the class $\mathcal{C}$, in the chosen EKR coordinates associated to $\mathcal{C}$ the bottom row in Sandwich Diagram has formally the same description as for the Cartan contact system on $J^{r}(1,2)$. In particular, because the relations (3) keep holding true in the vicinity of $\mathcal{C}$ in these coordinates, the covariant subdistribution $F$ of $D^{1}$ is there invariably of the form

$$
F=\left(\partial_{x^{i}}, \partial_{y^{i}} ; 1 \leq i \leq r\right)
$$

The symmetry $\mathcal{Y}$, preserving $\Delta^{r}=: D$, preserves the derived flag $\left(D^{j}\right)_{j=r}^{0}$ of $D$, so preserves this $F$, too. Hence the first three components of $\mathcal{Y}$ cannot depend on the variables $x^{i}$ and $y^{i}$ for $1 \leq i \leq r$, as stated in the lemma.

Remark 3. Note, however, one essential difference with the 1-flags in that here are three free functions in the base of the theory, instead of just one contact hamiltonian there (in formulas (8) ).

As previously, one needs some additional information about the code of $\mathcal{C}$. So for $j=2,3, \ldots, r$ we define

$$
s(j)= \begin{cases}0, & \text { when } i_{2}, \ldots, i_{j-1}=1, \\ \max \left\{s: 2 \leq s<j \& i_{s}>1\right\}, & \text { in the opposite case }\end{cases}
$$

Note that when $s(j) \geq 2$, then $i_{s(j)}=2$ or else $i_{s(j)}=3$. These two distinct (and disjoint) geometric situations account for bigger complexity of the recurrences to be produced. (The eventail of possible singularities of special 2-flags is much wider than for Goursat.) 


\section{Infinitesimal symmetries of special 2-flags got hold of}

Our main theorem of the paper, Theorem 2 below, shows that every infinitesimal symmetry is uniquely determined by the singularity class under consideration together with symmetry's first three component functions, denoted traditionally $A, B, C$, in an explicit, algorithmically computable manner. Namely,

Theorem 2 Let $U$ be the domain of EKR coordinates (14) chosen for an arbitrarily fixed singularity class $1 . i_{2} \cdot i_{3} \ldots i_{r}$. In those coordinates, all infinitesimal symmetries $\mathcal{Y}$ of $\Delta^{r}$ restricted to $U$ are of a particular form (17), where $A, B, C$ are free smooth functions of only $t, x^{0}, y^{0}$ and the $F^{s}, G^{s}, 1 \leq s \leq r$, are univocally recursively determined by $A, B, C$ and the class code, according to the formulae given in (20) and Lemmas 2, 3 and 4 below.

PROOF. We are going to ascertain one by one (or rather two by two) the consecutive components of vector fields $\mathcal{Y}$ in (17) above, from $F^{1}$ and $G^{1}$ on, given the initial arbitrary function data $A, B, C$. To this end we will use the truncations $\mathcal{Y}[j]$ of $\mathcal{Y}$ to the spaces of coordinates of indices $\leq j, j=1,2, \ldots, r$, on which the distributions $\Delta^{j}$ live:

$$
\mathcal{Y}[j]=A \partial_{t}+B \partial_{x^{0}}+C \partial_{y_{0}}+\sum_{s=1}^{j}\left(F^{s} \partial_{x^{s}}+G^{s} \partial_{y^{s}}\right) .
$$

Attention. The formulas (20) right below and in Lemmas 2, 3 and 4 below are, in the first place, only necessary for $\mathcal{Y}$ to be a true symmetry of $\Delta^{r}$. They became also sufficient in the last part of our (long) proof of Theorem 2

To begin with, let us demonstrate the argument on the 'baby' components $F^{1}$ and $G^{1}$. The infinitesimal invariance condition

$$
\left[\mathcal{Y}[1], \Delta^{1}\right] \subset \Delta^{1}
$$

clearly implies

$$
[\mathcal{Y}[1], Z[1]]=a_{1} Z[1]+b_{1} \partial_{x^{1}}+c_{1} \partial_{y^{1}}
$$

which in turn implies $a_{1}=-Z[1] A$. At the same time $F^{1}-Z B[1]=a_{1} x^{1}$ and $G^{1}-Z[1] C=a_{1} y^{1}$. Putting all this together,

$$
\left\{\begin{array}{l}
F^{1}=Z[1] B-x^{1} Z[1] A, \\
G^{1}=Z[1] C-y^{1} Z[1] A .
\end{array}\right.
$$

So indeed the pair of new components in $\mathcal{Y}[1]$ is univocally determined by the base components $A, B, C$. As for the coefficients $b_{1}$ and $c_{1}$ in (19), they get ascertained only after $F^{1}$ and $G^{1}$ are found.

This inference is an instance of a general 
Lemma 2 Assuming that an infinitesimal symmetry $\mathcal{Y}[j-1]$ of $\Delta^{j-1}$ is already known for certain $2 \leq j \leq r$, in the situation $i_{j}=1$, the $\partial_{x^{j}}-$ and $\partial_{y^{j}}-$ components of the prolongation $\mathcal{Y}[j]$ of $\mathcal{Y}[j-1]$ are as follows

$$
\begin{aligned}
& F^{j}= \begin{cases}Z[j] F^{j-1}-x^{j} Z[1] A, & \text { when } s(j)=0, \\
Z[j] F^{j-1}-x^{j} Z[s(j)] F^{s(j)-1}, & \text { when } s(j) \geq 2, i_{s(j)}=2, \\
Z[j] F^{j-1}-x^{j} Z[s(j)] G^{s(j)-1}, & \text { when } s(j) \geq 2, i_{s(j)}=3 .\end{cases} \\
& G^{j}= \begin{cases}Z[j] G^{j-1}-y^{j} Z[1] A, & \text { when } s(j)=0, \\
Z[j] G^{j-1}-y^{j} Z[s(j)] F^{s(j)-1}, & \text { when } s(j) \geq 2, i_{s(j)}=2, \\
Z[j] G^{j-1}-y^{j} Z[s(j)] G^{s(j)-1}, & \text { when } s(j) \geq 2, i_{s(j)}=3 .\end{cases}
\end{aligned}
$$

Proof of Lemma2 The vector field $\mathcal{Y}[j]$ infinitesimally preserves the distribution $\Delta^{j}$, whence

$$
[\mathcal{Y}[j], Z[j]]=a_{j} Z[j]+b_{j} \partial_{x^{j}}+c_{j} \partial_{y^{j}}
$$

for certain unspecified functions $a_{j}, b_{j}, c_{j}$. The coefficient $a_{j}$ is of central importance here. We typically work, here and in what will follow later, in the following order: - we firstly ascertain $a_{j}$, - secondly find (this is most important) $F^{j}$ and $G^{j}$, - eventually ascertain the values of $b_{j}$ and $c_{j}$.

The function $a_{j}$ can be extracted from (21) by watching this vector equation on the level of such a component of $Z[j]$ which is identically 1 . Inspecting the stepwise construction that leads from $Z[1]$ to $Z[j]$, there always is such a component! Namely, it is the $\partial_{t}$ - component when $s(j)=0$. When, on the contrary, $s(j) \geq 2$, it is either the $\partial_{x^{s(j)-1}}-$ component (when $i_{s(j)}=2$ ), or else it is the $\partial_{y^{s(j)-1}}-$ component (when $i_{s(j)}=3$ ). With thus specified information, it is a matter of course that

$$
a_{j}=- \begin{cases}Z[1] A, & \text { when } s(j)=0, \\ Z[s(j)] F^{s(j)-1}, & \text { when } s(j) \geq 2, i_{s(j)}=2, \\ Z[s(j)] G^{s(j)-1}, & \text { when } s(j) \geq 2, i_{s(j)}=3 .\end{cases}
$$

On the other hand, the same equation (21) watched on the level of $\partial_{x^{j-1}}$ reads

$$
F^{j}-Z[j] F^{j-1}=a_{j} x^{j},
$$

and watched on the level of $\partial_{y^{j-1}}$ reads

$$
G^{j}-Z[j] G^{j-1}=a_{j} y^{j} .
$$

The needed expressions for $F^{j}$ and $G^{j}$ follow upon substituting the expression (22) of $a_{j}$ into these two equations.

Lemma 3 Assuming that an infinitesimal symmetry $\mathcal{Y}[j-1]$ of $\Delta^{j-1}$ is already known for certain $2 \leq j \leq r$, in the situation $i_{j}=2$, the $\partial_{x^{j}}-$ and $\partial_{y^{j}}-$ components of the prolongation $\mathcal{Y}[j]$ of $\mathcal{Y}[j-1]$ are as follows

$$
F^{j}= \begin{cases}x^{j}\left(Z[1] A-Z[j] F^{j-1}\right), & \text { when } s(j)=0, \\ x^{j}\left(Z[s(j)] F^{s(j)-1}-Z[j] F^{j-1}\right), & \text { when } s(j) \geq 2, i_{s(j)}=2, \\ x^{j}\left(Z[s(j)] G^{s(j)-1}-Z[j] F^{j-1}\right), & \text { when } s(j) \geq 2, i_{s(j)}=3 .\end{cases}
$$




$$
G^{j}=Z[j] G^{j-1}-y^{j} Z[j] F^{j-1} .
$$

Proof of Lemma 3. The vector equation (21) still holds true. Now the $a_{j}$ coefficient can be (and easily) extracted from it at the level $\partial_{x^{j-1}}$, because the coefficient of the $\partial_{x^{j-1}}$ - component in $Z[j]$ is 1 :

$$
a_{j}=-Z[j] F^{j-1} .
$$

At the same time writing down the equal sides of (21) at the level $\partial_{y^{j-1}}$,

$$
G^{j}-Z[j] G^{j-1}=a_{j} y^{j},
$$

leads, by the way of (23), to the desired formula for $G^{j}$.

It is not that quick with the function $F^{j}$. It can be extracted from precisely one out of three levels of the $\partial_{t}-, \partial_{x^{s(j)-1}}-$, or $\partial_{y^{s(j)-1}}-$ components. Because one, once again, looks for a component in $Z[j]$ with a coefficient 1 , if 'enveloped' now in the factor $x^{j}$ (because $i_{j}>1$ in the proposition under proof).

In function of the position of that ' 1 ', equalling the relevant levels in (21), one gets precisely one relation out of the following three

$$
\begin{cases}F^{j}-x^{j} Z[1] A=a_{j} x^{j}, & \text { when } s(j)=0, \\ F^{j}-x^{j} Z[s(j)] F^{s(j)-1}=a_{j} x^{j}, & \text { when } s(j) \geq 2, i_{s(j)}=2, \\ F^{j}-x^{j} Z[s(j)] G^{s(j)-1}=a_{j} x^{j}, & \text { when } s(j) \geq 2, i_{s(j)}=3 .\end{cases}
$$

Then, accounting for (23), the desired formula for $F^{j}$ follows.

Lemma 4 Assuming that an infinitesimal symmetry $\mathcal{Y}[j-1]$ of $\Delta^{j-1}$ is already known for certain $2 \leq j \leq r$, in the situation $i_{j}=3$, the $\partial_{x^{j}}-$ and $\partial_{y^{j}}-$ components of the prolongation $\mathcal{Y}[j]$ of $\mathcal{Y}[j-1]$ are as follows

$$
\begin{gathered}
F^{j}= \begin{cases}x^{j}\left(Z[1] A-Z[j] G^{j-1}\right), & \text { when } s(j)=0, \\
x^{j}\left(Z[s(j)] F^{s(j)-1}-Z[j] G^{j-1}\right), & \text { when } s(j) \geq 2, i_{s(j)}=2, \\
x^{j}\left(Z[s(j)] G^{s(j)-1}-Z[j] G^{j-1}\right), & \text { when } s(j) \geq 2, i_{s(j)}=3 .\end{cases} \\
G^{j}=Z[j] F^{j-1}-y^{j} Z[j] G^{j-1} .
\end{gathered}
$$

Proof of Lemma 4. Invariably, the vector equation (21) keeps holding true. The $a_{j}$ coefficient on its right hand side can be extracted from it at the level $\partial_{y^{j-1}}$, because now the coefficient of the $\partial_{y^{j-1}}-$ component in $Z[j]$ is 1 :

$$
a_{j}=-Z[j] G^{j-1} .
$$

Then, writing simply down the equal sides of (21) at the level $\partial_{x^{j-1}}$,

$$
G^{j}-Z[j] F^{j-1}=a_{j} y^{j}
$$

leads, by the way of (24), to the presently needed formula for $G^{j}$. 
As for the function $F^{j}$, it can again be extracted from precisely one out of three levels of the $\partial_{t}-, \partial_{x^{s(j)-1}}-$, or $\partial_{y^{s(j)-1}}-$ components. In function of the position of that key component ' 1 ' in the field $Z[j]$, equalling the sides of the relevant levels in (21), one gets precisely one relation out of the following three

$$
\begin{cases}F^{j}-x^{j} Z[1] A=a_{j} x^{j}, & \text { when } s(j)=0, \\ F^{j}-x^{j} Z[s(j)] F^{s(j)-1}=a_{j} x^{j}, & \text { when } s(j) \geq 2, i_{s(j)}=2, \\ F^{j}-x^{j} Z[s(j)] G^{s(j)-1}=a_{j} x^{j}, & \text { when } s(j) \geq 2, i_{s(j)}=3 .\end{cases}
$$

Upon accounting for (24), the expected formula for $F^{j}$ follows.

As already invoiced, the obtained recursive formulas - at this moment only necessary - are also sufficient for the produced vector field $\mathcal{Y}$ to actually be a symmetry of $\Delta^{r}$. Indeed, knowing already that $[\mathcal{Y}, Z[r]] \in \Delta^{r}$ (cf. the always holding true formulas (21) taken now for $j=r$ ), what only remains to be done is to take the remaining two generators of $\Delta^{r}$ and justify the vector fields' inclusions

$$
\left[\mathcal{Y}, \partial_{x^{r}}\right], \quad\left[\mathcal{Y}, \partial_{y^{r}}\right] \in \Delta^{r}
$$

To that end we note that Lemma 1 coupled with formulas (20) and all those listed in auxiliary Lemmas 2, 3 and 4 yield by simple induction that, for $j=$ $1,2, \ldots, r$,

the components $F^{j}$ and $G^{j}$ of $\mathcal{Y}$ depend only on $t, x^{0}, y^{0}, x^{1}, y^{1}, \ldots, x^{j}, y^{j}$.

Using this information for $1 \leq j \leq r-1$ and again Lemma 1, one computes with ease

$$
\left[\mathcal{Y}, \partial_{x^{r}}\right]=-\left[\partial_{x^{r}}, \mathcal{Y}\right]=\left(-\partial_{x^{r}} F^{r}\right) \partial_{x^{r}}+\left(-\partial_{x^{r}} G^{r}\right) \partial_{y^{r}}
$$

and

$$
\left[\mathcal{Y}, \partial_{y^{r}}\right]=-\left[\partial_{y^{r}}, \mathcal{Y}\right]=\left(-\partial_{y^{r}} F^{r}\right) \partial_{x^{r}}+\left(-\partial_{y^{r}} G^{r}\right) \partial_{y^{r}}
$$

Now, at long last, the proof of Theorem 2 is complete.

\section{Applications of recursively computable infinites- imal symmetries to the local classification prob- lem}

The main motivation underlying the present contribution has been to advance results in the local classification problem for special 2-flags - to propose a late follow-up to the work [18. In fact, getting - recursively - hold of the infinitesimal symmetries of special 2-flag:77 opens a way to advance the local classification in lengths $r=5$ (cf. in this respect, in particular, section 7.2) and $r=6$ which have kept challenging the small monster community for the last 15 years (see the table preceding section 4.1).

\footnotetext{
7 and, as a matter of fact, of all special $m$-flags, $m \geq 2$, too - this being the subject of a possible another paper
} 


\subsection{Continuous modulus in the class 1.2.1.2.1.2.1}

Reiterating already, the exact local classification of special 2-flags (and, all the more so, all special multi-flags) in lengths exceeding 4 is, in its generality, unknown. It is not excluded that a continuous modulus of the local classification hides itself already somewhere in length 6 . Instead, we want to give an example in length 7 of the effectiveness of our formulae put forward in Section 6 .

A possibly deepest fact communicated in [18] was

Theorem 3 ([18]) In the singularity class $\mathcal{C}=1.2 .1 .2 .1 .2 .1$ of special 2-flags of length 7 there resides a continuous modulus of the local classification.

This was originally proved (in the year 2003, as a matter of fact) by brute force, and here is how the i.s.'s may help.

PROOF. In the coordinates constructed for the class $\mathcal{C}$ we work with certain germs of the distribution $\Delta^{7}$ which generates a locally universal special 2-flag of length 7 . The reference points for those germs belong to $\mathcal{C}$. More precisely, these are the points, say $P$, with the coordinates

$$
\begin{gathered}
t=x^{0}=y^{0}=x^{1}=y^{1}=x^{2}=y^{2}=0, \quad x^{3}=1, \\
y^{3}=x^{4}=y^{4}=0, \quad x^{5}=1, \quad y^{5}=x^{6}=y^{6}=0, \quad x^{7}=c, y^{7}=0 .
\end{gathered}
$$

We intend to infinitesimally move such $P$ only in the $\partial_{x^{7}}$ - direction. (Compare, for instance, [11, where also only the farthest part of a flag - Goursat in that occurrence - was subject to possible movies.) That is, we look for an i.s. having at a point $P$ of type (25) all but the $\partial_{x^{7}}-$ components vanishing. Remembering about the triangle pattern of dependence of those component functions, this means the vanishing of $A, B, C$ at $(0,0,0)$, the vanishing of $F^{j}\left(\pi_{7, j}(P)\right), G^{j}\left(\pi_{7, j}(P)\right)$ for $j=1,2, \ldots, 6$ and the vanishing of $G^{7}(P)$. The component $F^{7}(P)$ is not yet known and will be analyzed with care.

Initially we do not know how few/many such vector fields could exist. At any rate, any one of them is induced by certain functions $A, B, C$ in the variables $t, x, y$. The recurrence formulae are known from Section 6. When, among other components of an i.s., one wants to express $F^{7}(P)$ via those basic unknown functions $A, B, C$, one goes backwards along the code of $\mathcal{C}$, and firstly applies Lemma 2 (because $i_{7}=1$ ), then Lemma 3 (because $i_{6}=2$ ), then again Lemma 2 (because $i_{5}=1$ ), and so on intermittently. Upon applying with care these lemmas due numbers of times, the above-listed vanishings mean in the terms of the functions in the base

$$
\begin{aligned}
& 0=A(0,0,0)=B(0,0,0)=C(0,0,0)=\quad B_{t}(0,0,0)=C_{t}(0,0,0) \\
& =C_{x^{0}}(0,0,0)=c C_{t x^{0}}(0,0,0) \text {, }
\end{aligned}
$$

and - most important

$$
0=F^{3}\left(\pi_{7,3}(P)\right)=\left(3 A_{t}-2 B_{x^{0}}\right)(0,0,0)
$$




$$
0=F^{5}\left(\pi_{7,5}(P)\right)=\left(B_{x^{0}}-A_{t}\right)(0,0,0) .
$$

Now comes the punch line, because the outcome of the computations for $F^{7}$ is

$$
F^{7}(P)=3 c\left(A_{t}-B_{x^{0}}\right)(0,0,0) .
$$

Relations (26) and (27) together imply $A_{t}(0,0,0)=B_{x^{0}}(0,0,0)=0$. So $F^{7}(P)=0$ by (28). That is, every i.s. of $\mathcal{C}$ must infinitesimally freeze at $P$ the coordinate $x^{7}$, when it infinitesimally freezes all the remaining coordinates specified in (25). Theorem 3 is proved.

Remark 4. In other terms, the germs of the structure $\Delta^{7}$ at various points $P$ as above (i.e., for different values of the parameter $c$ ) are pairwise nonequivalent. The local geometry of the distribution $\Delta^{7}$ changes continuously within the discussed class $\mathcal{C}$.

\subsection{Towards the classification of the one step prolonga- tions within singularity class 1.2.1.1}

We conclude the paper by excerpting from [18 the partition, into the orbits of the local classification, of the singularity class 1.2.1.1 (when the width $m=2$, cf. Remark 5 on p. 37 there), and suggesting a line of possible continuation in the next length 5 . This class is not chosen at random; it splits into maximal (6) number of orbits in that length 4 , cf. Section 7 in [18]. The names of orbits are taken from that preprint. One means the germs of $\Delta^{4}$, watched in the EKR coordinates constructed for 1.2.1.1, at points, say $P$, having $t=x^{0}=y^{0}=$ $x^{1}=y^{1}=x^{2}=y^{2}=0$ and

\begin{tabular}{|c||c|c||c|c|}
\hline the orbit & $x^{3}\left(\pi_{4,3}(P)\right)$ & $y^{3}\left(\pi_{4,3}(P)\right)$ & $x^{4}(P)$ & $y^{4}(P)$ \\
\hline \hline $1.2 .1_{-\mathrm{s}, \text { tra }} \cdot 1$ & 1 & 0 & 0 & 0 \\
\hline $1.2 .1_{-\mathrm{s}, \tan .} 1_{-\mathrm{s}, \operatorname{tra}}$ & 0 & 1 & 1 & 0 \\
\hline $1.2 .1_{-\mathrm{s}, \tan \cdot 1_{-\mathrm{s}, \tan }}$ & 0 & 1 & 0 & 0 \\
\hline $1.2 .1_{+\mathrm{s}} \cdot 1_{-\mathrm{s}, \operatorname{tra}}$ & 0 & 0 & 1 & 0 \\
\hline $1.2 .1_{\mathrm{s}} \cdot 1_{-\mathrm{s}, \tan }$ & 0 & 0 & 0 & 1 \\
\hline $1.2 .1_{+\mathrm{s}}$ & 0 & 0 & 0 & 0 \\
\hline
\end{tabular}

Upon prolonging $\Delta^{4}$ to $\Delta^{5}$ in the vicinity of points of 1.2.1.1, one is to work with points in the classes 1.2.1.1. $i_{5}, i_{5} \in\{1,2,3\}$. The classification result recalled in the table above applies now to the distribution $\left[\Delta^{5}, \Delta^{5}\right]$ and as such remains true, regardless of the value of $i_{5}$ (the Lie square of $\Delta^{5}$ does not depend on new variables $\left.x^{5}, y^{5}\right)$. The same concerns the recursive formulae for the component functions $F^{j}, G^{j}, j=1,2,3,4$, of the i.s.'s of $\Delta^{5}$. Yet, naturally, expressions for the components $F^{5}, G^{5}$ depend critically on the value of $i_{5}$. Sticking to the points $P$ from the table, one is to analyze the expressions for $F^{5}(Q)$ and $G^{5}(Q)$, $Q \in$ 1.2.1.1. $i_{5}, \pi_{5,4}(Q)=P$. They are linear in $x^{5}(Q), y^{5}(Q)$, with coefficients depending on $P$ and on certain partials at $(0,0,0)$ of the basic functions $A, B, C$. 
All the difficulty resides in the - unknown and hard to compute - coefficients standing next to those partials.

An instructive example is given in section 7.1. The coefficient standing next to $c=x^{7}(P)$ on the RHS of (28) has appeared forced to be zero by the earlier infinitesimal normalizations (26) and (27). Because of that phenomenon, even the outcome of the classification of singularity class 1.2.1.1.1 $\left(i_{5}=1\right)$ is difficult to predict.

In general - in higher lengths - systems of coefficients in growing sets of partials of $A, B, C$ would play decisive roles in freezing or not of the values of new incoming pairs of component functions of the i.s.'s. Linear algebra packages would eventually come in handy.

\section{References}

[1] J. Adachi; Global stability of special multi-flags. Israel J. Math. 179, (2010), $29-56$.

[2] R. L. Bryant, L. Hsu; Rigidity of integral curves of rank 2 distributions. Invent. math. 114, 435-461.

[3] E. Cartan; Les systèmes de Pfaff à cinq variables et les équations aux dérivées partielles du second ordre. Ann. Ecole Normale 27 (1910), 109192.

[4] A. Castro, S. J. Colley, G. Kennedy, C. Shanbrom; A coarse stratification of the monster tower. Michigan Math. J. 66 (2017), 855-866.

[5] A. Castro, W. Howard; A Monster tower approach to Goursat multi-flags. Diff. Geom. Appl. 30 (2012), 405-427.

[6] A.Castro, R. Montgomery; Spatial curve singularities and the Monster/Semple tower. Israel J. Math. 192 (2012), 381-427.

[7] F. Jean; The car with $n$ trailers: characterization of the singular configurations. ESAIM: COCV 1 (1996), 241-266 (electronic).

[8] A. Kumpera, J. L. Rubin; Multi-flag systems and ordinary differential equations. Nagoya Math. J. 166 (2002), 1-27.

[9] A. Kumpera, C. Ruiz; Sur l'équivalence locale des systèmes de Pfaff en drapeau. In: "Monge-Ampère Equations and Related Topics", Rome 1982, 201-248.

[10] R. Montgomery, A Tour of Subriemannian Geometries, their Geodesics, and Applications. Math. Surveys Monographs 91, AMS (2002).

[11] R. Montgomery, M. Zhitomirskii; Geometric approach to Goursat flags. Ann. Inst. H. Poincaré (AN) 18 (2001), 459-493. 
[12] R. Montgomery, M. Zhitomirskii; Points and Curves in the Monster Tower. Memoirs AMS 956 (2010).

[13] P. Mormul; Goursat distributions with one singular hypersurface - constants important in their Kumpera-Ruiz pseudo-normal forms. Preprint No 185, Labo Topologie, Univ. de Bourgogne, Dijon (1999).

[14] P. Mormul; Contact hamiltonians distinguishing locally certain Goursat systems. Banach Center Publ. 51, Warsaw 2000, 219-230.

[15] P. Mormul; Geometric singularity classes for special $k$-flags, $k \geq 2$, of arbitrary length (2003). (https://www.mimuw.edu.pl/ mormul/Mor03.ps)

[16] P. Mormul; Multi-dimensional Cartan prolongation and special $k$-flags. Banach Center Publ. 65, Warsaw 2004, 157-178.

[17] P. Mormul; Singularity classes of special 2-flags. SIGMA 5 (2009), 102 (electronic).

[18] P. Mormul, F. Pelletier; Special 2-flags in lengths not exceeding four: a study in strong nilpotency of distributions; arXiv: 1011.1763 [math.DG] (2010).

[19] F. Pelletier, M.Slayman; Articulated arm and special multi-flags. J. Math. Sci. Adv. Appl. 8 (2011), 9-41.

[20] F. Pelletier, M. Slayman; Configurations of an articulated arm and singularities of special multi-flags. SIGMA 10 (2014), 059 (electronic).

[21] K. Shibuya, K. Yamaguchi; Drapeau theorem for differential systems. Diff. Geom. Appl. 27 (2009), 793-808.

[22] P. J.Vassiliou; A constructive generalised Goursat normal form. Diff. Geom. Appl. 24 (2006), 332-350.

[23] M.Zhitomirskii; Singularities and normal forms of smooth distributions. Banach Center Publ. 32, Warsaw 1993, 395-409.

Institute of MAthematics, University OF WARsaw

Banach str. 2, 02-097 Warsaw, Poland

E-MAIL ADDRESS: mormul@mimuw.edu.pl

Université de Savoie Mont Blanc (LAMA)

73370 Le Bourget-du-Lac, France

E-MAIL ADDRESS: fernand.pelletier@univ-smb.fr 